\title{
Creating anisotropic spin-split surface states in momentum space by molecular adsorption
}

\author{
Rico Friedrich, ${ }^{*}$ Vasile Caciuc, Bernd Zimmermann, Gustav Bihlmayer, Nicolae Atodiresei, ${ }^{\dagger}$ and Stefan Blügel \\ Peter Grünberg Institut (PGI-1) and Institute for Advanced Simulation (IAS-1), Forschungszentrum Jülich and JARA, \\ D-52425 Jülich, Germany
}

(Received 29 April 2017; published 2 August 2017)

\begin{abstract}
In this ab initio study we demonstrate that molecular adsorption on a surface Rashba system can be used to modulate the surface electronic structure in different momentum space directions, i.e., to create anisotropic spin splittings in $\mathbf{k}$ space. This effect is rooted in the asymmetric adsorption of the molecules on the surface in a hollow site which breaks the surface symmetry. More specifically, we demonstrate that the physisorbed $\mathrm{NH}_{3}$ has a small influence on the surface Rashba states and only gives rise to variations of the surface state Rashba parameters up to a factor of 1.4 over the surface Brillouin zone. In contrast, the chemisorption of $\mathrm{BH}_{3}$ leads to variations of the Rashba parameter by more than a factor of 2.5. Consequently, the anisotropy of the Rashba-split-surface states induced by molecular adsorption also gives rise to a modulation of the surface state spin texture, i.e., the out-of-plane spin polarization varies along different $\mathbf{k}$ directions by up to $70 \%$ for the occupied states. This offers the possibility to change the spin direction from in-plane to predominantly out-of-plane by modifying the electronic momentum by $90^{\circ}$.
\end{abstract}

DOI: 10.1103/PhysRevB.96.085403

\section{INTRODUCTION}

In modern solid state physics spin-orbit coupling (SOC) is of key importance for many intensively studied systems such as skyrmions [1], topological insulators [2], and Rashba materials [3]. In terms of spintronics especially the Rashba effect offered new avenues based, e.g., on the spin fieldeffect transistor suggested by Datta and Das in which spin control is achieved by electric fields [4] as recently realized experimentally [5].

The Bychkov-Rashba effect [6-8] initially investigated for semiconductor heterostructures describes the SOC induced lifting of the spin degeneracy in momentum space due to a structurally inversion asymmetric potential within a twodimensional electron gas (2DEG). In a simple model it can be described as the spin splitting arising from the asymmetric potential acting as an effective magnetic field in the frame of the moving electron [9]. The degree of spin splitting can then be characterized by the parameter $k_{\mathrm{SO}}$ indicating the shift of the extremum of the energy dispersion away from the degeneracy point present without SOC.

Besides for semiconducting materials the Rashba effect was also observed in case of surface states with the first experimental evidence found for the $\mathrm{Au}(111)$ surface [10] revealing a spin splitting of $k_{\mathrm{SO}} \approx 0.012 \AA^{-1}$. In the ongoing research intensive efforts had been undertaken to tune and enlarge the size of this Rashba spin splitting. For the low index surfaces of clean Bi [11] or surface alloys of $\mathrm{Bi}[12,13]$ or $\mathrm{Pb}[13,14]$ spin splittings have been reported exceeding the one of $\mathrm{Au}(111)$ by about one order of magnitude. Also a stoichiometry change of the surface alloy [15], graphene coverage [16,17], and the adsorption of adatoms [18-22] and

\footnotetext{
*Present address: Center for Materials Genomics, Department of Mechanical Engineering and Materials Science, Duke University, Durham, North Carolina 27708, USA; rico.friedrich@duke.edu, r.friedrich@fz-juelich.de

${ }^{\dagger}$ n.atodiresei@fz-juelich.de
}

organic molecules $[23,24]$ have been applied to investigate this issue.

In a complementary approach we have recently demonstrated that inorganic molecules can be employed to wind the surface state spin texture from in-plane to out-of-plane by inducing molecule specific in-plane surface potential gradients [25].

However, from another perspective many studies in the last years focused on the possibility to realize an anisotropic Rashba effect, i.e., Rashba-like surface states for which the spin splitting and band dispersion are different along different directions of the 2D surface Brillouin zone (BZ). It is important to note that for 2DEGs such an anisotropic Rashba spin splitting of electronic states can lead to specific features of the optical conductivity as compared with those obtained from the isotropic Rashba effect [26].

One way such an anisotropy can be induced is by the interference of structure inversion asymmetry (Rashba like) and bulk inversion asymmetry (Dresselhaus like) [27,28]. In addition, based on a group theoretical analysis of the surface symmetry, in Ref. [29] it was outlined that an anisotropic Rashba splitting should be expected for surfaces with, e.g., $C_{2 v}$ symmetry such as $\mathrm{Au}(110)$. Relativistic firstprinciples calculations indeed confirmed that the dispersion of surface states of differently reconstructed $\mathrm{Au}(110)$ surfaces is anisotropic [30,31]. This behavior was outlined to be a consequence of the admixture of bulk states of different symmetry to the surface state [31]. Recently, an anisotropic Rashba effect was also considered for deep $d$-type surface states [32], in monolayer black phosphorus [33], for a hydrogenated $\mathrm{ZnO}(10 \overline{10})$ surface [34] and in model systems based on an anisotropic two-dimensional electron gas [35].

Moreover, in order to explain the intrinsic anisotropy of the surface states of the $\mathrm{BiAg}_{2} / \mathrm{Ag}(111)$ system $\left(C_{3 v}\right.$ symmetry) third order terms in the wave number in the Hamiltonian were proposed [36]. These concepts were then also applied to rationalize the dispersion behavior of the Dirac-cone-like surface state at the W(110) surface [37] and in transition metal dichalcogenide monolayers upon application of a strong 
electric field [38]. Remarkably, in Refs. [39,40] not only an anisotropic dispersion of a surface state of the Tl/Si(111) system was found but in addition the spin texture was outlined to be anisotropic. Recently, the importance of a low surface symmetry that stabilizes an antiskyrmionic magnetic structure on 2 ML Fe on W(110) through the anisotropic DzyaloshinskiiMoriya interaction was also emphasized [41].

In this contribution we demonstrate from first principles that also the adsorption of inorganic molecules can be used to modulate the Rashba splitting of surface states along different momentum space directions. Moreover, the molecular adsorption allows us to change the spin-splitting character of an unoccupied surface state from non-Rashba-like to Rashbalike along specific $\mathbf{k}$ directions. Overall, these effects are intrinsically related to the reduction of the surface symmetry upon molecular adsorption.

More specifically, we investigate two inorganic molecules ammonia $\left(\mathrm{NH}_{3}\right)$ and borane $\left(\mathrm{BH}_{3}\right)$ on the $\mathrm{BiAg} / \mathrm{Ag}(111)$ surface alloy. While $\mathrm{NH}_{3}$ contains an electronegative nitrogen atom with a free electron pair, $\mathrm{BH}_{3}$ incorporates an electropositive $\mathrm{B}$ atom with an empty $p_{z}$ orbital. The physisorption of $\mathrm{NH}_{3}$ on $\mathrm{BiAg}_{2} / \mathrm{Ag}(111)$ causes only minor changes of the surface state band structure but nevertheless gives rise to a modulation of the Rashba parameters up to a factor of 1.4 over the surface BZ. Even more strikingly, the chemisorption of $\mathrm{BH}_{3}$ creates a strong anisotropy in the Rashba splitting of the surface states and thereby causes a variation of the Rashba parameters larger than a factor of 2.5. Eventually, this leads to an out-of-plane spin polarization that varies by up to $70 \%$ along different $\mathbf{k}$ directions. Hence the $\mathbf{k}$-space anisotropy induced by the molecular adsorption also translates into a modulation of the spin texture along different directions in the surface BZ which is a fingerprint feature of the molecular orientation on the surface.

\section{COMPUTATIONAL DETAILS}

Electronic structure calculations were performed in the density functional theory (DFT) framework [42,43] making use of the VASP program [44-46] employing the same computational setup as described in Ref. [25]. Note that all molecule-surface configurations considered in our study were relaxed using the rev-vdW-DF2 functional in a spinunpolarized calculation [47-51]. However, at variance with the setup reported in Ref. [25], in this study we employed a ten layer asymmetric slab, i.e., the molecular layer is adsorbed only on one side of the slab. The band structures were then calculated for this setup using the PBE functional including $\mathrm{SOC}$ as this functional has been shown to reliably describe the electronic properties of the $\mathrm{BiAg}_{2} / \mathrm{Ag}(111)$ surface alloy [13]. Within these calculations both the $\overline{\Gamma M}$ as well as the $\overline{\Gamma K}$ directions were each sampled by $100 k$ points. For the constant energy cuts a symmetric slab was used to avoid artifacts arising from the Ag surface state of the asymmetric slab and the whole surface BZ was sampled by $100 \times 100 k$ points.

To obtain the effective mass of the surface states, the surface state band structure was fitted quadratically in a $\mathbf{k}$ range away from $\bar{\Gamma}$ up to the $\mathbf{k}$ vector for which the energy of the surface state coincides with the one at the crossing point at $\bar{\Gamma}$. Only in case of the occupied surface state this range was reduced to about $0.15 \AA^{-1}$ around $\bar{\Gamma}$ into the respective $\mathbf{k}$ direction since for larger ranges the dispersion was not quadratic anymore. According to the Rashba model the Rashba parameter $\alpha_{\mathrm{R}}$ is related to the Rashba momentum splitting $k_{\text {SO }}$ by

$$
\alpha_{\mathrm{R}}=\hbar^{2} k_{\mathrm{SO}} / m^{*},
$$

where $\hbar$ is Planck's constant divided by $2 \pi$ and $m^{*}$ is the effective mass of the considered surface states. The Rashba energy $E_{\mathrm{R}}$ is then expressed as

$$
E_{\mathrm{R}}=\hbar^{2} k_{\mathrm{SO}}^{2} / 2 m^{*} \text {. }
$$

\section{RESULTS AND DISCUSSION}

An overview of the ground state adsorption positions of both $\mathrm{NH}_{3}$ and $\mathrm{BH}_{3}$ on the $\mathrm{BiAg}_{2} / \mathrm{Ag}(111)$ surface alloy is given in Fig. 1. The $\mathrm{N}$ of the physisorbed $\mathrm{NH}_{3}$ adsorbs in an fcc hollow position, whereas the $\mathrm{B}$ of the weakly chemisorbed $\mathrm{BH}_{3}$ adsorbs in an hcp hollow site as outlined in Ref. [25]. Consequently, the adsorption pattern of the molecules on the surface leads to a symmetry reduction with respect to the clean surface. To clearly underline this point and its significance for the band structure of the hybrid molecule-surface systems, in Fig. 1, in addition to the real space surface unit cell, also the surface BZ is sketched on top of the structures together with the basis vectors of the reciprocal lattice.

To analyze the electronic structure of the molecule-surface systems considered in our ab initio study, the key starting point is to note that as a consequence of the adsorption of the two molecules the hexagonal symmetry of the uppermost surface layer is broken (the overall symmetry is reduced from $C_{3 v}$ to $\left.C_{s}\right)$. According to Fig. 1, the equivalence of all six $\overline{\Gamma M}$ as well as all six $\overline{\Gamma K}$ directions, present for the clean surface
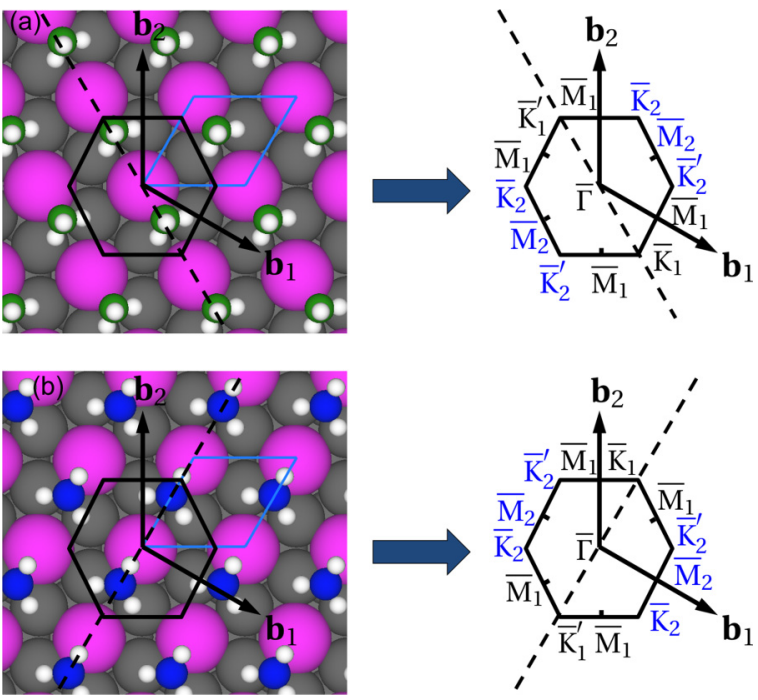

FIG. 1. Ground state geometries with surface BZs for (a) $\mathrm{NH}_{3}$ and (b) $\mathrm{BH}_{3}$ on the $\mathrm{BiAg}_{2} / \mathrm{Ag}(111)$ surface alloy (left side). The adsorption of the molecules breaks the sixfold rotational symmetry of the surface layer. Hence the high-symmetry points of the surface BZ become inequivalent (right side). The real space unit cell of each system is marked in light blue and the dashed line indicates the direction along which the $\mathrm{NH}_{3}$ and $\mathrm{BH}_{3}$ molecules are aligned, respectively. $\mathbf{b}_{1}$ and $\mathbf{b}_{2}$ stand for the basis vectors of the reciprocal lattice. Color code: Bi (magenta), Ag (dark gray), N (green), B (blue), and $\mathrm{H}$ (light gray). 

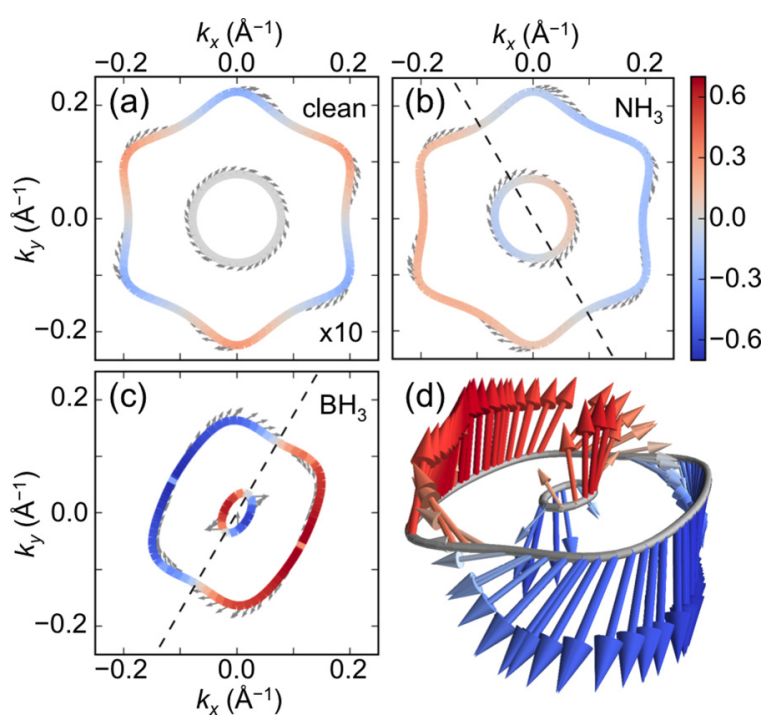

FIG. 2. Constant energy contours at $0.1 \mathrm{eV}$ above the Fermi energy for (a) the clean $\mathrm{BiAg}_{2} / \mathrm{Ag}(111)$ surface alloy, (b) for $\mathrm{NH}_{3}-\mathrm{BiAg}_{2} / \mathrm{Ag}(111)$, and (c) for $\mathrm{BH}_{3}-\mathrm{BiAg}_{2} / \mathrm{Ag}(111)$. In each case the direction of the in-plane spin polarization is denoted by arrows and the magnitude of the out-of-plane spin polarization is indicated by the color scale. Note that in case of the clean $\mathrm{BiAg}_{2} / \mathrm{Ag}(111)$ surface alloy the out-of-plane spin polarization was magnified by a factor 10. (d) Perspective view of the constant energy contour for the $\mathrm{BH}_{3}-\mathrm{BiAg}_{2} / \mathrm{Ag}(111)$ system with a $3 \mathrm{D}$ vector representation of the spin texture.

alloy, is removed. In each case the molecules are positioned along a specific $\overline{\Gamma K}_{1}$ direction which reflects the remaining mirror symmetry element of the system. The other corners of the hexagonal surface BZ are labeled by $\bar{K}_{2}$ [52]. Similarly, the four $\bar{M}$ points close to the $\overline{\Gamma K}_{1}$ direction are indicated as $\bar{M}_{1}$, whereas the others are denoted by $\bar{M}_{2}$. Thus for each of the two hybrid systems the band structure will be depicted along four $\mathbf{k}$ directions, namely $\overline{\Gamma M}_{1}, \overline{\Gamma K}_{1}, \overline{\Gamma M}_{2}$, and $\overline{\Gamma K}_{2}$.

\section{A. Surface state energy dispersions}

To first clearly show the effect of the symmetry breaking onto the surface state energy dispersion induced by the molecular adsorption, we present in Fig. 2 a constant energy cut at $0.1 \mathrm{eV}$ above the Fermi energy for each system. For the clean surface in Fig. 2(a) the cut resembles the behavior known from the literature [12]. In case of the $\mathrm{NH}_{3}$ based hybrid system [Fig. 2(b)] the shape of the cut is similar to the one of the clean surface. However, the $z$ (out-of-plane) component of the spin polarization clearly shows the symmetry reduction revealing only a twofold symmetry. For the $\mathrm{BH}_{3}-\mathrm{BiAg}_{2} / \mathrm{Ag}(111)$ system [Fig. 2(c)] also the shape of the constant energy contour clearly shows the twofold symmetry and in addition an especially strong out-of-plane spin polarization.

To discuss the behavior now in more detail, the band structures of the hybrid systems are presented in Fig. 3 with the Rashba parameters that give a direct measure for the strength of the spin splitting listed in Table I (The Rashba splittings and Rashba energies are reported in Tables II and III). For the
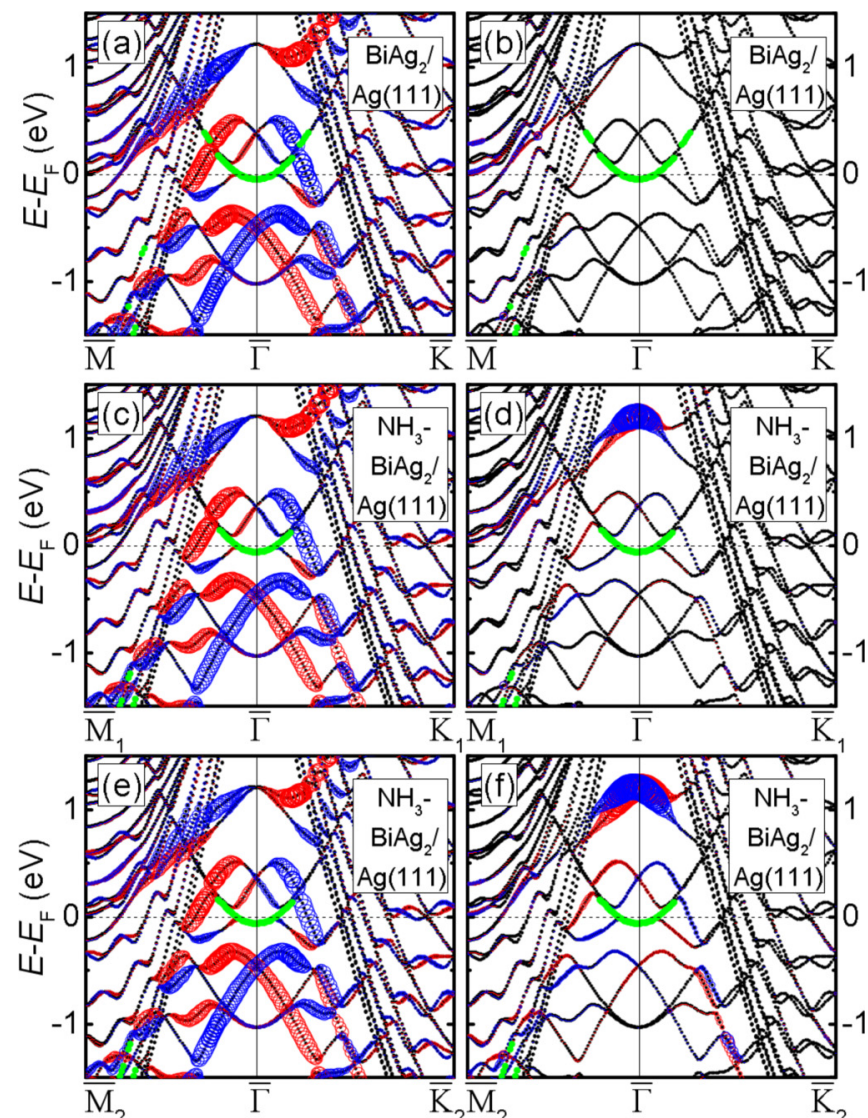

$\mathrm{K}_{1} \mathrm{M}$

\section{.}
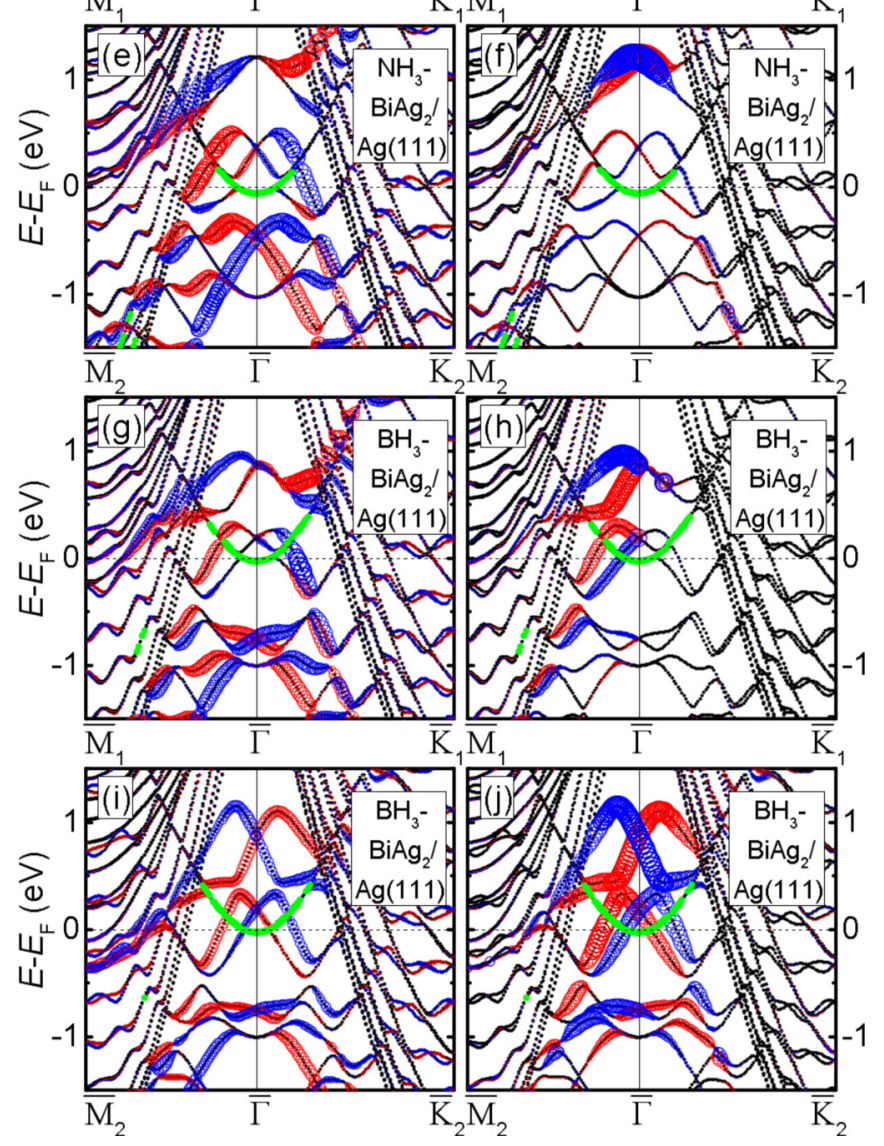

FIG. 3. Band structures with in-plane [(a), (c), (e), (g), and (i)] and out-of-plane [(b), (d), (f), (h), and (j)] spin polarization of (a) and (b) the clean $\mathrm{BiAg}_{2} / \mathrm{Ag}(111)$ surface alloy, (c)-(f) for $\mathrm{NH}_{3}-\mathrm{BiAg}_{2} / \mathrm{Ag}(111)$, and (g)-(j) for $\mathrm{BH}_{3}-\mathrm{BiAg}_{2} / \mathrm{Ag}(111)$. The size of the symbols is proportional to the degree of spin polarization indicating positive values by red and negative values by blue larger than $2 \%$. In case of the in-plane spin polarization the spin quantization axis is aligned perpendicular to both the surface normal as well as the respective in-plane $\mathbf{k}$ direction [13]. States localized at the clean $\operatorname{Ag}(111)$ surface side of the asymmetric slab are marked in green. 
TABLE I. Rashba parameters $\alpha_{\mathrm{R}}^{\text {occ }}, \alpha_{\mathrm{R}}^{\text {unocc }}$, and $\alpha_{\mathrm{R}}^{\text {unocc, } 2}$ of the Rashba split surface states for the clean surface alloy and $\mathrm{NH}_{3}$ as well as $\mathrm{BH}_{3}$ on $\mathrm{BiAg}_{2} / \operatorname{Ag}(111)$.

\begin{tabular}{|c|c|c|c|c|c|c|c|c|c|c|c|c|}
\hline \multirow[b]{2}{*}{ System/surf. } & \multicolumn{4}{|c|}{$\alpha_{\mathrm{R}}^{\mathrm{occ}}(\mathrm{eV} \AA)$} & \multicolumn{4}{|c|}{$\alpha_{\mathrm{R}}^{\mathrm{unocc}}(\mathrm{eV} \AA)$} & \multicolumn{4}{|c|}{$\alpha_{\mathrm{R}}^{\text {unocc, }, 2}(\mathrm{eV} \AA)$} \\
\hline & $\overline{\Gamma M}_{1}$ & $\overline{\Gamma K}_{1}$ & $\overline{\Gamma M}_{2}$ & $\overline{\Gamma K}_{2}$ & $\overline{\Gamma M}_{1}$ & $\overline{\Gamma K}_{1}$ & $\overline{\Gamma M}_{2}$ & $\overline{\Gamma K}_{2}$ & $\overline{\Gamma M}_{1}$ & $\overline{\Gamma K}_{1}$ & $\overline{\Gamma M}_{2}$ & $\overline{\Gamma K}_{2}$ \\
\hline Clean surf. & -2.48 & -2.50 & -2.48 & -2.50 & -3.43 & -3.99 & -3.43 & -3.99 & - & - & - & - \\
\hline $\mathrm{NH}_{3}$ & -2.63 & -2.54 & -2.90 & -2.76 & -3.21 & -3.81 & -3.92 & -4.51 & - & - & - & - \\
\hline $\mathrm{BH}_{3}$ & - & - & - & - & -3.65 & -2.31 & -5.93 & -5.08 & -2.43 & - & -6.24 & -4.29 \\
\hline
\end{tabular}

clean $\mathrm{BiAg}_{2} / \mathrm{Ag}(111)$ surface alloy [see Figs. 3(a) and 3(b)] there are three surface states in the considered energy range close to $\bar{\Gamma}$, one occupied Rashba split state, a first unoccupied Rashba split state, and the second unoccupied state split by higher orders in $k$ [53].

We first focus on the dispersion of the electronic states of the molecule-surface systems and will later on refer to their spin texture. For the $\mathrm{NH}_{3}$ based hybrid system [see Figs. 3(c)-3(f)] the molecular adsorption induces only small modifications in the surface state band structure meaning that the dispersion along the different $\mathbf{k}$ directions shows only minor differences with respect to each other and compared to the clean surface case [see Figs. 3(a) and 3(b)]. Nevertheless, the Rashba splitting of the occupied surface state is slightly increased compared to the clean surface (cs) value of $k_{\mathrm{SO}}^{\mathrm{cs}, \text { occ }}=0.09 \AA^{-1}$ to $k_{\mathrm{SO}}^{\mathrm{NH}_{3} \text {,occ }}=0.10 \AA^{-1}$ in all $\mathbf{k}$ directions as pointed out in Ref. [25]. This is consistent with the increased Rashba parameters of $\alpha_{\mathrm{R}}^{\mathrm{NH}_{3}, \text { occ }}=-2.54$ to $-2.90 \mathrm{eV} \AA$ as compared to the clean surface reference values of $\alpha_{\mathrm{R}}^{\mathrm{cs}, \text { occ }}=-2.48 /-2.50 \mathrm{eV} \AA$ along $\overline{\Gamma M} / \overline{\Gamma K}$, respectively (see Table I).

In addition, the splitting of the unoccupied Rashba split surface state becomes slightly anisotropic in $\mathbf{k}$ space, i.e., $k_{\mathrm{SO}}^{\mathrm{NH}_{3} \text {, unocc }}=0.09 \AA^{-1}$ along $\overline{\Gamma M}_{2}$, whereas it coincides with the clean surface value of $0.08 \AA^{-1}$ along the other directions. Consistently, a small variation of the Rashba parameter of the order of $1.30 \mathrm{eV} / \AA$ (a factor of 1.4) over the surface BZ is found (see $\overline{\Gamma M}_{1}$ vs $\overline{\Gamma K}_{2}$ in Table I). Note that this anisotropy is significantly larger than the intrinsic anisotropy of the Rashba parameter of this surface state, i.e., for the clean surface, being of the order of $0.56 \mathrm{eV} / \AA$ (a factor of 1.2) over the surface BZ.

In case of the $\mathrm{BH}_{3}$ based hybrid system [see Figs. 3(g)-3(j)] the band structures do not directly indicate a Rashba splitting of the occupied surface state along all $\mathbf{k}$ directions. This surface state becomes a surface resonance since it has been pushed into the bulk Ag band structure [25,54]. This behavior can be deduced from the finding that the occupied surface state has finite contributions over all layers of the slab and is hence not localized at the surface.

More importantly, the first unoccupied surface state shows a Rashba-like splitting which is strongly anisotropic along different $\mathbf{k}$ directions, i.e., $k_{\mathrm{SO}}^{\mathrm{BH}_{3} \text {, unocc }}=0.09 / 0.07 \AA^{-1}$ along $\overline{\Gamma M}_{1} / \overline{\Gamma M}_{2}$, whereas it persists at $0.08 \AA^{-1}$ along $\overline{\Gamma K}_{1}$ and $\overline{\Gamma K}_{2}$. As a consequence, the corresponding Rashba parameter $(-2.31 \mathrm{eV} \AA)$ and Rashba energy $(-0.07 \mathrm{eV})$ are small along $\overline{\Gamma K}_{1}$, whereas an increase with respect to the clean surface values of $-3.43 /-3.99 \mathrm{eV} \AA$ and $-0.12 /-0.12 \mathrm{eV}$ to $-5.93 /-5.08 \mathrm{eV} \AA$ and $-0.14 /-0.14 \mathrm{eV}$ is observed along $\overline{\Gamma M}_{2} / \overline{\Gamma K}_{2}$. Thus the maximum of the parabolic energy dispersion of this surface state is modulated on a scale of $70 \mathrm{meV}$ along the different $\mathbf{k}$ directions.

Remarkably, the second unoccupied surface state [see Figs. 3(g) and 3(i)] shows now also a Rashba-like splitting [25] which is however only present along $\overline{\Gamma M}_{1}, \overline{\Gamma M}_{2}$, and $\overline{\Gamma K}_{2}$ but vanishes along $\overline{\Gamma K}_{1}$, the alignment direction of the molecules indicated in Fig. 1(b). The induced splitting ranges from $k_{\mathrm{SO}}^{\mathrm{BH}_{3}, \text { unocc, } 2}=0.07$ to $0.09 \AA^{-1}$ and is therefore of the same size as for the aforementioned first unoccupied split surface state. Due to the small effective mass of this surface state along $\overline{\Gamma M}_{2}$ and $\overline{\Gamma K}_{2}$, the corresponding Rashba parameters and Rashba energies amount to -6.24 and $-4.29 \mathrm{eV} \AA$ as well as -0.28 and $-0.23 \mathrm{eV}$, respectively, and thereby exceed the values of all other surface states considered in this study.

A rationalization of this induced splitting can be given when the orbital contributions of this second unoccupied electronic state at $\bar{\Gamma}$ with respect to the surface layer atoms are analyzed. For the clean surface these are $s: 0 \%, p_{x}$ : $32.6 \%, p_{y}: 32.6 \%$, and $p_{z}: 0 \%$. For the $\mathrm{BH}_{3}-\mathrm{BiAg}_{2} / \mathrm{Ag}(111)$ hybrid system these are modified to $s: 4.5 \%, p_{x}: 35.8 \%$, $p_{y}: 20.8 \%$, and $p_{z}: 3.3 \%$, i.e., the state becomes polarized by $s$ and $p_{z}$ character at the expense of the $p_{y}$ character due to the molecular adsorption which is a key ingredient to obtain a sizable Rashba splitting $[9,55]$. We note that

TABLE II. Spin-splitting parameters of the occupied $k_{\mathrm{SO}}^{\text {occ }}$ and unoccupied $k_{\mathrm{SO}}^{\text {unocc }} / k_{\mathrm{SO}}^{\text {unocc, } 2}$ Rashba split surface states for $\mathrm{NH}_{3} / \mathrm{BH}_{3}$ on the $\mathrm{BiAg}_{2} / \mathrm{Ag}(111)$ surface alloy.

\begin{tabular}{|c|c|c|c|c|c|c|c|c|c|c|c|c|}
\hline \multirow[b]{2}{*}{ System/surf. } & \multicolumn{4}{|c|}{$k_{\mathrm{SO}}^{\mathrm{occ}}\left(\AA^{-1}\right)$} & \multicolumn{4}{|c|}{$k_{\mathrm{SO}}^{\text {unocc }}\left(\AA^{-1}\right)$} & \multicolumn{4}{|c|}{$k_{\mathrm{SO}}^{\text {unocc }, 2}\left(\AA^{-1}\right)$} \\
\hline & $\overline{\Gamma M}_{1}$ & $\overline{\Gamma K}_{1}$ & $\overline{\Gamma M}_{2}$ & $\overline{\Gamma K}_{2}$ & $\overline{\Gamma M}_{1}$ & $\overline{\Gamma K}_{1}$ & $\overline{\Gamma M}_{2}$ & $\overline{\Gamma K}_{2}$ & $\overline{\Gamma M}_{1}$ & $\overline{\Gamma K}_{1}$ & $\overline{\Gamma M}_{2}$ & $\overline{\Gamma K}_{2}$ \\
\hline Clean surf. & 0.09 & 0.09 & 0.09 & 0.09 & 0.08 & 0.08 & 0.08 & 0.08 & - & - & - & - \\
\hline $\mathrm{NH}_{3}$ & 0.10 & 0.10 & 0.10 & 0.10 & 0.08 & 0.08 & 0.09 & 0.08 & - & - & - & - \\
\hline $\mathrm{BH}_{3}$ & - & - & - & - & 0.09 & 0.08 & 0.07 & 0.08 & 0.07 & - & 0.09 & 0.09 \\
\hline
\end{tabular}


TABLE III. Rashba energies $E_{\mathrm{R}}^{\text {occ }}, E_{\mathrm{R}}^{\text {unocc }}$, and $E_{\mathrm{R}}^{\text {unocc, } 2}$ of the Rashba split surface states for $\mathrm{NH}_{3} / \mathrm{BH}_{3}$ on the $\mathrm{BiAg} 2 / \mathrm{Ag}(111)$ surface alloy.

\begin{tabular}{|c|c|c|c|c|c|c|c|c|c|c|c|c|}
\hline \multirow[b]{2}{*}{ System/surf. } & \multicolumn{4}{|c|}{$E_{\mathrm{R}}^{\mathrm{occ}}(\mathrm{eV})$} & \multicolumn{4}{|c|}{$E_{\mathrm{R}}^{\text {unocc }}(\mathrm{eV})$} & \multicolumn{4}{|c|}{$E_{\mathrm{R}}^{\mathrm{unocc}, 2}(\mathrm{eV})$} \\
\hline & $\overline{\Gamma M}_{1}$ & $\overline{\Gamma K}_{1}$ & $\overline{\Gamma M}_{2}$ & $\overline{\Gamma K}_{2}$ & $\overline{\Gamma M}_{1}$ & $\overline{\Gamma K}_{1}$ & $\overline{\Gamma M}_{2}$ & $\overline{\Gamma K}_{2}$ & $\overline{\Gamma M}_{1}$ & $\overline{\Gamma K}_{1}$ & $\overline{\Gamma M}_{2}$ & $\overline{\Gamma K}_{2}$ \\
\hline Clean surf. & -0.11 & -0.11 & -0.11 & -0.11 & -0.12 & -0.12 & -0.12 & -0.12 & - & - & - & - \\
\hline $\mathrm{NH}_{3}$ & -0.13 & -0.13 & -0.13 & -0.13 & -0.12 & -0.11 & -0.14 & -0.13 & - & - & - & - \\
\hline $\mathrm{BH}_{3}$ & - & - & - & - & -0.11 & -0.07 & -0.14 & -0.14 & -0.09 & - & -0.28 & -0.23 \\
\hline
\end{tabular}

the remaining contributions to the state are localized in the lower Ag layers. Furthermore, the strong in-plane potential gradients induced by the molecular adsorption also play a crucial role. As outlined in Refs. [12,56], both in-plane and out-of-plane potential gradients are important to obtain large Rashba splittings.

Hence the $\mathrm{BH}_{3}$ adsorption on the $\mathrm{BiAg}_{2} / \mathrm{Ag}(111)$ surface alloy allows us to create a new Rashba-split surface state along specific $\mathbf{k}$ directions which are determined by the particular adsorption pattern of this molecule. Moreover, for both unoccupied surface states the Rashba parameter varies by a factor of more than 2.5 over the surface BZ. In Refs. [31-34] similar variations of the Rashba parameter in the momentum space ranging up to a factor of 11 have been reported. However, in our case the absolute value of the Rahba parameters and therefore the general strength of the Rashba effect is at least about an order of magnitude higher. We expect that these features of the unoccupied surface states should be accessible by experimental techniques such as inverse angle-resolved photoelectron spectroscopy or two-photon photoemission spectroscopy.

For the $\mathrm{BH}_{3}$-based hybrid system the chemical bonding of this molecule to the surface plays a crucial role for its electronic structure. This chemical contribution of the $\mathrm{BH}_{3}$ can be directly visualized by the charge density of the individual surface states in the planes marked as dashed lines in Fig. 1 for each system as indicated in Fig. 4. More specifically, in Figs. 4(a)-4(c) for the clean surface the $\left(s, p_{z}\right)$-like character of the occupied Rashba split surface state as well as the $p_{(x, y)}$-like character of the two unoccupied surface states at $\bar{\Gamma}$ can be clearly identified. For the $\mathrm{NH}_{3}$-based hybrid system [Figs. 4(d)-4(f)] these surface states are only slightly distorted and practically no orbital contribution at the molecular site is visible. On the contrary, in case of the $\mathrm{BH}_{3}$-based hybrid system [Figs. 4(g)-4(i)] the molecule clearly couples to the Bi-derived surface states, i.e., it binds to the surface states. This bonding effect is strongest in case of the occupied surface state since the orbital contribution at the molecular site in Fig. 4(g) is clearly largest compared to the other two. This indicates that the strong coupling of the molecule to this state contributes to the shift of this state into the bulk Ag bands as seen in the band structures above. In addition, in Figs. 4(j)-4(1) the charge density of the surface states of the $\mathrm{BH}_{3}-\mathrm{BiAg}_{2} / \mathrm{Ag}(111)$ system is depicted in top view from which the twofold symmetry due to molecular adsorption can be clearly seen.

\section{B. Surface state spin textures}

From a spintronics viewpoint it is now crucial to investigate the effect of the molecular adsorption onto the spin texture of the Rashba spin-split states. As we recently demonstrated in Ref. [25], the strong in-plane potential gradients induced by the chemisorption of inorganic molecules such as $\mathrm{BH}_{3}$ turn the spin polarization predominantly out of the surface plane in contrast to the case of the clean surface where the spin polarization lies essentially within the surface plane $[12,13]$. Here we focus now on the consequences of the molecular induced k-space anisotropy for the spin texture of the surface states.

In Figs. 3(a), 3(c) 3(e), 3(g), and 3(i) the in-plane spin polarization of each electronic state is plotted on top of the band structure for both the clean surface [Fig. 3(a)] as well as for the hybrid systems along the different $\mathbf{k}$ directions [Figs. 3(c), 3(e) 3(g), and 3(i)]. For the $\mathrm{NH}_{3}$-based hybrid system [see Figs. 3(c) and 3(e)] the differences between the spin polarization obtained for different $\mathbf{k}$ directions and to that of the clean surface are small. Compared to the
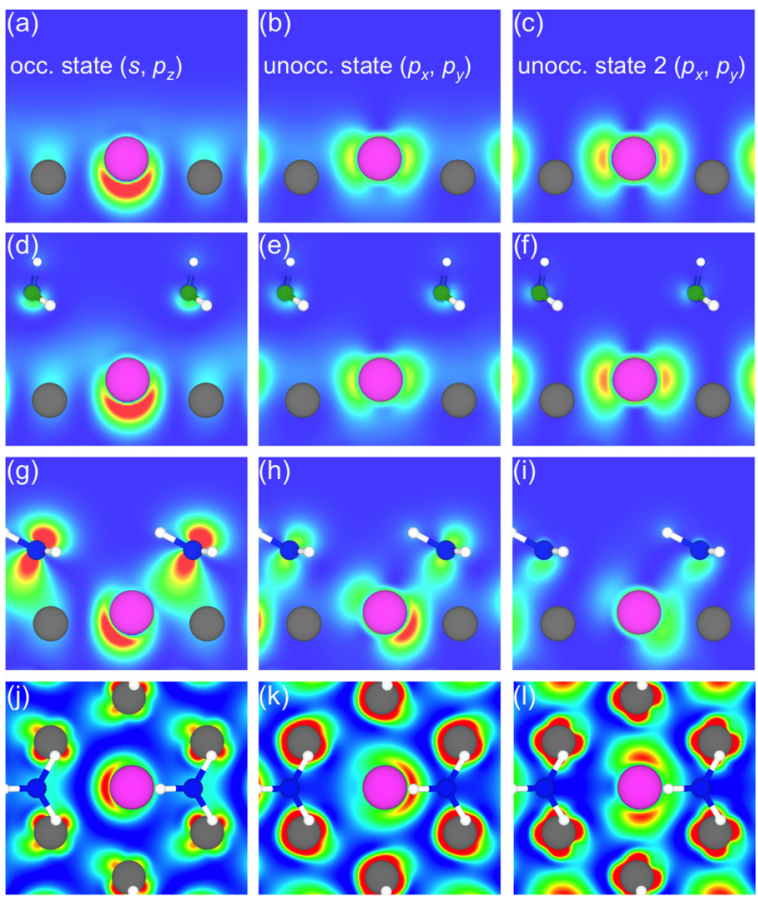

0

$6.75 \cdot 10^{-2} e / \AA^{3}$

FIG. 4. Visualization of the charge density of the occupied and unoccupied surface states at $\bar{\Gamma}$ for (a)-(c) the clean $\mathrm{BiAg}_{2} / \mathrm{Ag}(111)$ surface alloy, (d)-(f) for $\mathrm{NH}_{3}-\mathrm{BiAg}_{2} / \mathrm{Ag}(111)$, and (g)-(i) for $\mathrm{BH}_{3}-\mathrm{BiAg}_{2} / \mathrm{Ag}(111)$ in the planes marked as dashed lines in Fig. 1, respectively. (j)-(l) Top view of the charge density of the surface states for the $\mathrm{BH}_{3}-\mathrm{BiAg}_{2} / \mathrm{Ag}(111)$ system clearly indicating the twofold symmetry. 
clean $\mathrm{BiAg}_{2} / \mathrm{Ag}(111)$ surface only a slight reduction of the maximum in-plane spin polarization from about $70 \%$ to about $65 \%$ for the occupied states is observed. However in the $\mathrm{BH}_{3}$-based hybrid system [see Figs. 3(g) and 3(i)] clearly the anisotropy of the electronic states in the different $\mathbf{k}$ directions also leads to an anisotropic spin polarization [57]. More specifically, the in-plane spin polarization is reduced compared to the clean surface case and the maximum spin polarization of the occupied states reaches about $60 \%$ (predominantly along $\left.\overline{\Gamma K}_{1}\right)$ and 50\% in Figs. 3(g) and 3(i), respectively.

Complementary, the out-of-plane spin polarization of the states is visualized in Figs. 3(b), 3(d) 3(f), 3(h), and 3(j). For the $\mathrm{NH}_{3}$-based hybrid system in Fig. 3(d) as in case of the clean surface [see Fig. 3(b)] practically no out-of-plane spin polarization is found along the $\overline{\Gamma K}_{1}$ direction. This is a consequence of the fact that the $\mathrm{NH}_{3}$ molecule lies along this direction (see Fig. 1) representing a mirror symmetry element of the system. Hence no in-plane potential gradients perpendicular to this direction can occur and therefore no outof-plane spin polarization is observed along $\overline{\Gamma K}_{1}$. However, in the other three $\mathbf{k}$ directions this argument does not hold and therefore finite in-plane potential gradients are induced by the $\mathrm{NH}_{3}$ adsorption which lead to sizable out-of-plane spin polarization contributions reaching up to about $20 \%$ for the occupied states in Fig. 3(f). This is qualitatively different from the case of the clean surface where a sizable out-of-plane spin polarization was only reported close to the edge of the bulk projected Ag band gap [12,58].

Interestingly, an exception to this behavior is observed in case of the second unoccupied surface state around $\bar{\Gamma}$ showing strong out-of-plane spin polarization. This indicates that the increased outward buckling of the surface $\mathrm{Bi}$ due to $\mathrm{NH}_{3}$ adsorption [25] enhances the atomiclike nature of the spin polarization of this $p_{(x, y)}$ derived state favoring out-of-plane contributions.

Even more remarkable is the case of the $\mathrm{BH}_{3}$-based hybrid system in Figs. 3(h) and 3(j). Again the band structure along $\overline{\Gamma K}_{1}$ shows no sizable polarization since along this direction a symmetry plane is present in this system. However for the other $\mathbf{k}$ directions strong out-of-plane spin polarizations reaching up to about $50 \%$ and $70 \%$ in case of the occupied states in Figs. 3(h) and 3(j), respectively, are observed. This means that the in-plane potential gradients induced by the $\mathrm{BH}_{3}$ adsorption onto the $\mathrm{BiAg}_{2} / \mathrm{Ag}(111)$ surface alloy are large enough to induce out-of-plane spin polarizations even exceeding the corresponding in-plane contributions [25]. In addition, the strong variation of the spin polarization direction between different $\mathbf{k}$ directions allows us to switch the spin direction from in-plane along $\overline{\Gamma K}_{1}$ to predominantly out-of-plane along $\overline{\Gamma M}_{2}$ being perpendicular to $\overline{\Gamma K}_{1}$ (see Fig. 1).

This k-space anisotropy of the spin polarization direction induced by the molecular adsorption can be most clearly seen in Fig. 2 from the constant energy cuts with the spin polarization contributions depicted on top. For clarity, only the direction of the in-plane spin polarization is indicated by the arrows in Figs. 2(a)-2(c). As a general observation, it is important to note that the constant energy contours reported in Fig. 2 reveal a lowering of the $C_{3 v}$ symmetry of the spin polarization for the clean $\mathrm{BiAg}_{2} / \mathrm{Ag}(111)$ surface [see Fig. 2(a)] to a $C_{s}$ one for the $\mathrm{NH}_{3}-$ and $\mathrm{BH}_{3}-\mathrm{BiAg}_{2} / \mathrm{Ag}(111)$

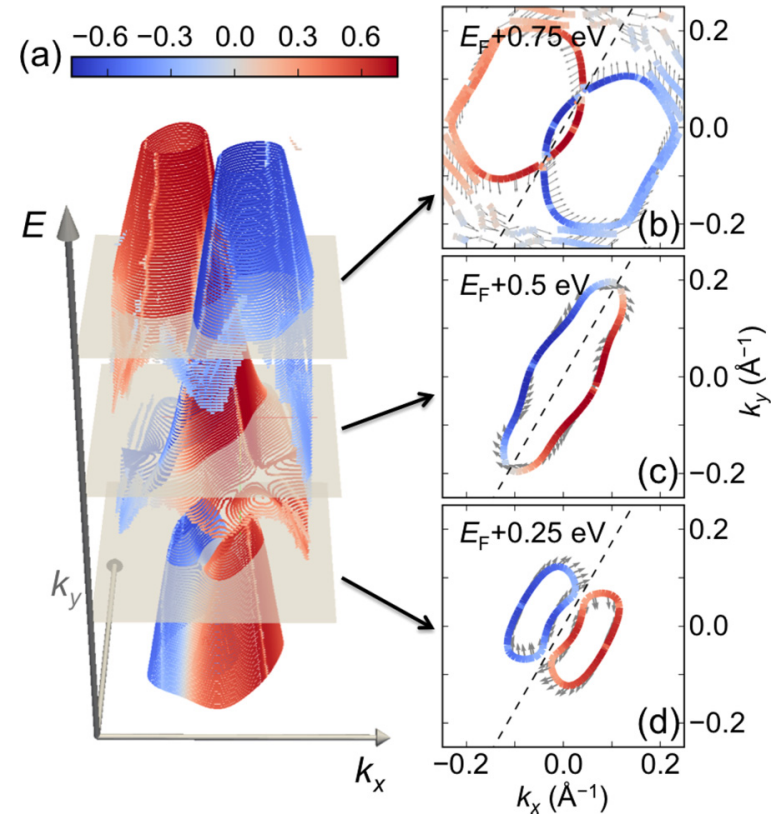

FIG. 5. (a) Band structure of the surface states for the $\mathrm{BH}_{3}-\mathrm{BiAg}_{2} / \mathrm{Ag}(111)$ system: The inner part of the Brillouin zone $\left(\left|k_{x, y}\right|<0.25 \AA^{-1}\right)$ is shown in an energy range $[0,1 \mathrm{eV}]$ relative to the Fermi energy. (b)-(d) Constant-energy cuts at energies of $0.25,0.5$, and $0.75 \mathrm{eV}$ above the Fermi energy, respectively. The out-of-plane component of the spin polarization is indicated by the color. Only states are shown whose charge is localized in the upper surface layer by more than (a) $30 \%$, (b) $20 \%$, or (c) and (d) $50 \%$.

systems [see Figs. 2(b) and 2(c), respectively]. Now, as regarding the spin texture of these Rashba spin-split states, we note that the magnitude of the in-plane spin polarization for the inner circle is about $20 \%$, whereas it amounts to about $70 \%$ for the outer part of the energy contour for both the clean $\mathrm{BiAg}_{2} / \mathrm{Ag}(111)$ surface alloy as well as for the $\mathrm{NH}_{3}-\mathrm{BiAg}_{2} / \mathrm{Ag}(111)$ system. However, the range of the out-of-plane and in-plane spin polarization contributions over the surface $\mathrm{BZ}$ in case of the $\mathrm{BH}_{3}-\mathrm{BiAg}_{2} / \mathrm{Ag}(111)$ system is more complex and therefore we also visualize this spin texture in a perspective view in Fig. 2(d). In this figure the strong variation of the spin direction with the change in momentum direction becomes evident.

To outline this special behavior of the $\mathrm{BH}_{3}-\mathrm{BiAg}_{2} / \mathrm{Ag}(111)$ system in more detail for a larger energy range we present in Fig. 5 the band structure of the surface states in an energy range of $[0,1 \mathrm{eV}]$ above the Fermi energy with three additional representative energy cuts. There it can be clearly seen that in each case the shape of the contour deviates significantly from the circular dispersion revealed by the Rashba model and that there is a strongly energy-dependent anisotropy of both the dispersion and the spin polarization.

These results clearly illustrate how the anisotropy in momentum space induced by the asymmetric molecular adsorption which breaks the surface symmetry carries over to an associated anisotropic spin texture. Importantly, this $k$-space anisotropy of the spin texture induced by the molecular adsorption for the hybrid $\mathrm{NH}_{3}-$ and $\mathrm{BH}_{3}-\mathrm{BiAg}_{2} / \mathrm{Ag}(111)$ systems is present for both physisorbed $\left(\mathrm{NH}_{3}\right)$ as well as 
chemisorbed $\left(\mathrm{BH}_{3}\right)$ molecules, i.e., the spin texture of the surface states in $\mathbf{k}$ space is a fingerprint feature of the molecular adsorption pattern on the surface. These findings are especially interesting in view of the fact that the Fermi level could be shifted to higher/lower energies by $\mathrm{Te} / \mathrm{Pb}$ doping.

\section{CONCLUSIONS}

In this $a b$ initio study we investigated how the adsorption of two inorganic molecules ammonia $\left(\mathrm{NH}_{3}\right)$ and borane $\left(\mathrm{BH}_{3}\right)$ leads to an anisotropy in the band structure of the Rashba split surface states of the $\mathrm{BiAg}_{2} / \mathrm{Ag}(111)$ surface alloy, i.e., it creates a variation of the Rashba splitting along different directions in momentum space. The origin of this anisotropy lies in the asymmetric adsorption of the molecules in a hollow site of the surface which breaks the surface symmetry. Specifically, we find that the physisorbed $\mathrm{NH}_{3}$ molecule has only a minor effect on the Rashba state band structure but still causes a variation of the Rashba parameters up to a factor 1.4 over the surface BZ. Conversely, the chemisorbed $\mathrm{BH}_{3}$ molecule gives rise to strong variations of the Rashba splittings and Rashba state dispersions and hence leads to a modulation of the Rashba parameters of larger than a factor of 2.5 over the surface BZ. Moreover, it modifies the spin splitting of an unoccupied surface state from third order in $k$ to Rashba like (linear order in $k$ ) along specific $\mathbf{k}$ directions.

Importantly, the molecules also create an anisotropic spin texture in momentum space. Although the variation of the out-of-plane spin polarization contributions of the occupied states in case of the $\mathrm{NH}_{3}$ based hybrid system are small $(\leqslant 20 \%)$ along different $\mathbf{k}$ directions, $\mathrm{BH}_{3}$ adsorption allows for variations as large as $70 \%$ between different directions.
Based on this behavior it can be envisioned that the spin direction of surface state electrons in this latter system can be switched from in-plane to predominantly out-of-plane by changing the electronic momentum by $90^{\circ}$. Based on these findings our results present an interesting direction in spintronics research in which the spin polarization in devices could be widely tuned upon adsorption of nonmagnetic inorganic molecules to engineer their optical and transport properties.

\section{ACKNOWLEDGMENTS}

S.B., N.A., and V.C. gratefully acknowledge financial support from the Volkswagen-Stiftung through the "Optically Controlled Spin Logic" project and by the Deutsche Forschungsgemeinschaft (DFG) through the Collaborative Research Center SFB 1238 (Project C01). The authors gratefully acknowledge the Gauss Centre for Supercomputing (GCS) for providing computing time through the John von Neumann Institute for Computing (NIC) on the GCS share of the supercomputer JUQUEEN [59] at Jülich Supercomputing Centre (JSC). GCS is the alliance of the three national supercomputing centres HLRS (Universität Stuttgart), JSC (Forschungszentrum Jülich), and LRZ (Bayerische Akademie der Wissenschaften), funded by the German Federal Ministry of Education and Research (BMBF) and the German State Ministries for Research of Baden-Württemberg (MWK), Bayern (StMWFK) and Nordrhein-Westfalen (MIWF). The authors gratefully acknowledge the computing time granted by the JARA-HPC Vergabegremium and VSR commission on the supercomputer JUROPA/JURECA [60] at Forschungszentrum Jülich.
[1] S. Heinze, K. von Bergmann, M. Menzel, J. Brede, A. Kubetzka, R. Wiesendanger, G. Bihlmayer, and S. Blügel, Nat. Phys. 7, 713 (2011).

[2] M. Z. Hasan and C. L. Kane, Rev. Mod. Phys. 82, 3045 (2010).

[3] A. Manchon, H. C. Koo, J. Nitta, S. M. Frolov, and R. A. Duine, Nat. Mater. 14, 871 (2015).

[4] S. Datta and B. Das, Appl. Phys. Lett. 56, 665 (1990).

[5] P. Chuang, S.-C. Ho, L. W. Smith, F. Sfigakis, M. Pepper, C.-H. Chen, J.-C. Fan, J. P. Griffiths, I. Farrer, H. E. Beere, G. A. C. Jones, D. A. Ritchie, and T.-M. Chen, Nat. Nanotechnol. 10, 35 (2015).

[6] E. I. Rashba, Soviet Phys. Solid State 2, 1109 (1960).

[7] Y. A. Bychkov and E. I. Rashba, J. Phys. C 17, 6039 (1984).

[8] Y. A. Bychkov and E. I. Rashba, JETP Lett. 39, 78 (1984).

[9] M. Heide, G. Bihlmayer, P. Mavropoulos, A. Bringer, and S. Blügel, Newslett. Psi-K Network 78 (2006), http://www.psik.org/newsletters/News_78/Highlight_78.pdf.

[10] S. LaShell, B. A. McDougall, and E. Jensen, Phys. Rev. Lett. 77, 3419 (1996).

[11] Y. M. Koroteev, G. Bihlmayer, J. E. Gayone, E. V. Chulkov, S. Blügel, P. M. Echenique, and Ph. Hofmann, Phys. Rev. Lett. 93, 046403 (2004).

[12] C. R. Ast, J. Henk, A. Ernst, L. Moreschini, M. C. Falub, D. Pacilé, P. Bruno, K. Kern, and M. Grioni, Phys. Rev. Lett. 98, 186807 (2007).
[13] G. Bihlmayer, S. Blügel, and E. V. Chulkov, Phys. Rev. B 75, 195414 (2007).

[14] D. Pacilé, C. R. Ast, M. Papagno, C. Da Silva, L. Moreschini, M. Falub, A. P. Seitsonen, and M. Grioni, Phys. Rev. B 73, 245429 (2006).

[15] C. R. Ast, D. Pacilé, L. Moreschini, M. C. Falub, M. Papagno, K. Kern, M. Grioni, J. Henk, A. Ernst, S. Ostanin, and P. Bruno, Phys. Rev. B 77, 081407(R) (2008).

[16] A. Varykhalov, D. Marchenko, M. R. Scholz, E. D. L. Rienks, T. K. Kim, G. Bihlmayer, J. Sanchez-Barriga, and O. Rader, Phys. Rev. Lett. 108, 066804 (2012).

[17] J. Sánchez-Barriga, G. Bihlmayer, D. Wortmann, D. Marchenko, O. Rader, and A. Varykhalov, New J. Phys. 15, 115009 (2013).

[18] E. Rotenberg, J. W. Chung, and S. D. Kevan, Phys. Rev. Lett. 82, 4066 (1999).

[19] M. Hochstrasser, J. G. Tobin, E. Rotenberg, and S. D. Kevan, Phys. Rev. Lett. 89, 216802 (2002).

[20] F. Forster, S. Hüfner, and F. Reinert, J. Phys. Chem. B 108, 14692 (2004).

[21] O. Krupin, G. Bihlmayer, K. Starke, S. Gorovikov, J. E. Prieto, K. Döbrich, S. Blügel, and G. Kaindl, Phys. Rev. B 71, 201403(R) (2005).

[22] L. Moreschini, A. Bendounan, C. R. Ast, F. Reinert, M. Falub, and M. Grioni, Phys. Rev. B 77, 115407 (2008). 
[23] M. C. Cottin, J. Lobo-Checa, J. Schaffert, C. A. Bobisch, R. Möller, J. E. Ortega, and A. L. Walter, New J. Phys. 16, 045002 (2014).

[24] B. Stadtmüller, J. Seidel, N. Haag, L. Grad, C. Tusche, G. van Straaten, M. Franke, J. Kirschner, C. Kumpf, M. Cinchetti, and M. Aeschlimann, Phys. Rev. Lett. 117, 096805 (2016).

[25] R. Friedrich, V. Caciuc, G. Bihlmayer, N. Atodiresei, and S. Blügel, New J. Phys. 19, 043017 (2017).

[26] A. Mawrie and T. K. Ghosh, J. Phys.: Condens. Matter. 28, 425302 (2016).

[27] S. D. Ganichev and W. Prettl, J. Phys.: Condens. Matter 15, R935 (2003).

[28] S. Giglberger, L. E. Golub, V. V. Bel'kov, S. N. Danilov, D. Schuh, C. Gerl, F. Rohlfing, J. Stahl, W. Wegscheider, D. Weiss, W. Prettl, and S. D. Ganichev, Phys. Rev. B 75, 035327 (2007).

[29] T. Oguchi and T. Shishidou, J. Phys.: Condens. Matter 21, 092001 (2009).

[30] M. Nagano, A. Kodama, T. Shishidou, and T. Oguchi, J. Phys.: Condens. Matter 21, 064239 (2009).

[31] E. Simon, A. Szilva, B. Ujfalussy, B. Lazarovits, G. Zarand, and L. Szunyogh, Phys. Rev. B 81, 235438 (2010).

[32] R. Requist, P. M. Sheverdyaeva, P. Moras, S. K. Mahatha, C. Carbone, and E. Tosatti, Phys. Rev. B 91, 045432 (2015).

[33] Z. S. Popović, J. M. Kurdestany, and S. Satpathy, Phys. Rev. B 92, 035135 (2015).

[34] M. A. U. Absor, F. Ishii, H. Kotaka, and M. Saito, AIP Adv. 6, 025309 (2016).

[35] A. Johansson, J. Henk, and I. Mertig, Phys. Rev. B 93, 195440 (2016).

[36] S. Vajna, E. Simon, A. Szilva, K. Palotas, B. Ujfalussy, and L. Szunyogh, Phys. Rev. B 85, 075404 (2012).

[37] K. Miyamoto, A. Kimura, T. Okuda, K. Shimada, H. Iwasawa, H. Hayashi, H. Namatame, M. Taniguchi, and M. Donath, Phys. Rev. B 86, 161411(R) (2012).

[38] C. Cheng, J.-T. Sun, X.-R. Chen, H.-X. Fu, and S. Meng, Nanoscale 8, 17854 (2016).

[39] S. D. Stolwijk, K. Sakamoto, A. B. Schmidt, P. Krüger, and M. Donath, Phys. Rev. B 90, 161109(R) (2014).

[40] S. D. Stolwijk, K. Sakamoto, A. B. Schmidt, P. Krüger, and M. Donath, Phys. Rev. B 91, 245420 (2015).
[41] M. Hoffmann, B. Zimmermann, G. P. Müller, D. Schürhoff, N. S. Kiselev, C. Melcher, and S. Blügel, Nat. Commun., doi:10.1038/s41467-017-00313-0 [arXiv:1702.07573].

[42] P. Hohenberg and W. Kohn, Phys. Rev. 136, B864 (1964).

[43] W. Kohn and L. J. Sham, Phys. Rev. 140, A1133 (1965).

[44] G. Kresse and J. Hafner, Phys. Rev. B 49, 14251 (1994).

[45] G. Kresse and J. Furthmüller, Phys. Rev. B 54, 11169 (1996).

[46] G. Kresse and J. Furthmüller, Comput. Mater. Sci. 6, 15 (1996).

[47] M. Dion, H. Rydberg, E. Schröder, D. C. Langreth, and B. I. Lundqvist, Phys. Rev. Lett. 92, 246401 (2004).

[48] K. Lee, E. D. Murray, L. Kong, B. I. Lundqvist, and D. C. Langreth, Phys. Rev. B 82, 081101(R) (2010).

[49] J. Klimeš, D. R. Bowler, and A. Michaelides, J. Phys.: Condens. Matter. 22, 022201 (2010).

[50] J. Klimeš, D. R. Bowler, and A. Michaelides, Phys. Rev. B 83, 195131 (2011).

[51] I. Hamada, Phys. Rev. B 89, 121103(R) (2014).

[52] Note that $\bar{K}_{2}$ and $\bar{K}_{1}$ are in principle equivalent due to the translational symmetry in $\mathbf{k}$ space and we denoted the translational inequivalent $\bar{K}$ points by additional primes. The $\overline{\Gamma K}_{1}$ direction is however not equivalent to $\overline{\Gamma K}_{2}$.

[53] R. Winkler, Spin-Orbit Coupling Effects in Two-Dimensional Electron and Hole Systems, Springer Tracts in Modern Physics, Vol. 191 (Springer, Berlin, 2003).

[54] Due to the fact that the considered slab consisted of ten layers, the bulk Ag band structure is reduced to quantum well states and one should therefore take into account that for a half infinite system more bulk bands will be present in the energy region of the surface resonance.

[55] G. Bihlmayer, Y. M. Koroteev, P. M. Echenique, E. V. Chulkov, and S. Blügel, Surf. Sci. 600, 3888 (2006).

[56] J. Premper, M. Trautmann, J. Henk, and P. Bruno, Phys. Rev. B 76, 073310 (2007).

[57] We note that although the spin polarization is anisotropic over the surface BZ, no net magnetization is acquired in the systems since due to Kramer's degeneracy opposite directions in $\mathbf{k}$ space have opposite spin polarization.

[58] F. Meier, H. Dil, J. Lobo-Checa, L. Patthey, and J. Osterwalder, Phys. Rev. B 77, 165431 (2008).

[59] M. Stephan and J. Docter, JLSRF 1, 1 (2015).

[60] D. Krause and P. Thörnig, JLSRF 2, 62 (2016). 